\title{
Cuando el lenguaje excluye: consideraciones sobre el lenguaje no binario indirecto
}

Ártemis López

Doctorande en Estudios Lingüísticos

Universidade de Vigo

\section{Resumen}

Aunque las personas no binarias existimos en diversas culturas desde hace siglos, es un hecho indiscutible que nuestra visibilidad y representación está aumentando en todos los ámbitos. Con este aumento vienen retos lingüísticos para las personas que no forman parte de nuestra comunidad pero que quieren o deben hablar de nosotres, como reporteres o traductores que, con frecuencia, reproducen información sin tener contacto directo con los sujetos de ese discurso. Esta falta de familiaridad puede repercutir de manera negativa en obras cuyo proceso creativo incluyó documentación extensa y puede dejar al margen a las personas no binarias que formen parte del público objetivo. Este artículo trata varios casos de traducciones problemáticas o erróneas relacionadas con el lenguaje no binario indirecto (LNI) para ilustrar los problemas que también supone el LNI en la comunicación, traducida o no.

Palabras clave: género; lenguaje inclusivo; lenguaje no binario; traducción; transgénero

\begin{abstract}
Although non-binary people have existed in a variety of cultures for centuries, it is an unquestionable fact that the visibility and representation of our community is increasing in all fields. This increase brings with it linguistic challenges for those outside our community but who want to or have to speak about us - this could include reporters or translators, who frequently reproduce information without having direct contact with the subjects of that discourse. This lack of familiarity can have a negative effect on works that included thorough documentation as part of the creative process and can leave out non-binary members of the target audience. The present article goes over several cases of problematic or erroneous translations related to indirect non-binary language (INL) in order to showcase the problems that INL can pose in communication, translated or not.
\end{abstract}

Keywords: gender; inclusive language; non-binary language; translation; transgender

\section{El español no binario}

La lengua española se divide, tradicionalmente, en dos géneros gramaticales: el femenino, frecuentemente representado por el morfema $\{-\mathrm{a}\}$, y el género que suele expresarse mediante 
los morfemas $\{-\emptyset\}$ y $\{-o\}$ y que, según la norma, cumple las funciones de un género masculino y de uno genérico. Las dimensiones genérica y masculina expresadas generalmente por los morfemas $\{-\emptyset /-0\}$ no siempre se pueden distinguir en la práctica, especialmente en determinados contextos discursivos, y pueden llegar a ser exclusivamente masculinas en algunos sociolectos. En ciertos contextos, el uso del llamado «masculino genérico» puede ir en contra de la situación comunicativa, como serían un congreso de mujeres en la ciencia o el reciente artículo del periódico $A B C$ sobre la ministra Petra De Sutter, que originalmente la llamaba «el primer ministro transexual de Europa» (Serbeto 2020) y cambió su titular tras múltiples protestas de la comunidad (Los Replicantes 2020). Más allá de estas situaciones colectivas y genéricas, el uso de un género gramatical para hablar de una persona no binaria concreta merece consideración. Así como «María Méndez y Mercedes Bengoechea son dos científicos españoles» constituye un uso claramente erróneo y no genérico, hablar en masculino de cualquier persona que no utilice ese género gramatical también lo es.

El lenguaje no binario es solo una pequeña parte del «lenguaje inclusivo», que también incluye estrategias lingüísticas antirracistas, anticapacitistas y anticoloniales, entre muchas otras. Para hablar con precisión del lenguaje no binario, es preciso dividirlo en dos: lenguaje no binario directo e indirecto (López 2019a, 2019b). El lenguaje no binario indirecto, o LNI, tiene como objetivo hacer referencia a todos los géneros sin emplear marcas de género. Para ello, emplea ciertas estrategias lingüísticas como utilizar participios en lugar de adjetivos («le ha sorprendido» en lugar de «está sorprendido») o el uso de epicenos («el pueblo argentino» o «las personas argentinas» en lugar de «los argentinos»). Estas estrategias, por lo general, son difíciles de detectar o demostrar, pues se camuflan en la norma. El lenguaje no binario 
directo, o LND, es mucho más obvio porque utiliza neologismos y neomorfemas como $\{$-e $\}$ $\mathrm{o}\{-\mathrm{x}\}$ («está sorprendide» $\mathrm{y} \ll$ les argentines», para los ejemplos anteriores).

La visibilidad del LND lo convierte en blanco de burlas y en sujeto frecuente de discusión, mientras que el LNI permanece prácticamente invisible tanto para sus adversaries como para sus simpatizantes. No obstante, ambas categorías son igualmente importantes y merecen que les profesionales se familiaricen con ellas pues, aunque comparten la voluntad de romper la concepción genérica del masculino, las dos categorías trasladan mensajes radicalmente diferentes. El LND comunica de manera inequívoca que le autore respeta y apoya a las personas no binarias, mientras que el LNI depende de la situación: puede utilizarse para evitar el masculino genérico en una traducción, pero también puede ocultar las identidades no binarias por aversión e irreverencia o por miedo a la reacción del público.

El LNI podría parecer la mejor opción para incluir a todas las personas, pero es un arma de doble filo en cuanto a las personas no binarias. Les receptores suelen pasar el LNI por alto si no lo están buscando de manera activa y su uso puede provocar malentendidos. Además, como sucedió tras la elección de la Delegada Danica Roem en Virginia (Gilchrist 2017), el LNI se puede utilizar por razones tránsfobas para negar la identidad de las personas trans y no binarias. Si bien el LNI es perfectamente inclusivo en contextos genéricos y mixtos, su uso reiterado para hablar de personas que han expresado su género gramatical puede dar la impresión de negar su identidad.

Para ilustrar algunos de los problemas que pueden surgir de un uso descuidado del LNI, presento algunos estudios de caso a continuación. Primero hablaré en detalle sobre la traducción de Your Magic Mind and Body, un libro ubicado en el mundo de Steven Universe, y después de las traducciones de personajes concretos de tres series de televisión: Theo en 
Chilling Adventures of Sabrina, Double Trouble en She-Ra y God y Polution en Good Omens. ${ }^{1}$

\section{La traducción de le receptore}

Steven Universe es una serie de animación creada por Rebecca Sugar, une autore no binarie de Estados Unidos. En el universo de Steven Universe, los seres humanos viven junto a las Gemas, seres extraterrestres que usan los pronombres she/her en inglés, aunque el guion deja claro que todas las gemas son seres no binarios, con cuerpos hechos de luz y sin órganos, con lo que no necesitan ni comer ni dormir. Más adelante en la serie aparece un personaje, Stevonnie, que es la combinación de un personaje masculino y otro femenino con lo que Stevonnie es intersexual (Ermac 2019). Todo esto se establece en la serie sin problemas por parte de los personajes, pues es una serie acerca de la autoaceptación, el respeto mutuo, el amor y la amistad. A lo largo de la serie, varios personajes se enfrentan a desafíos diversos, ya sean batallas intergalácticas, como en «Reunited» (episodio especial de la temporada 5), o sentimientos de culpabilidad como en «Mindful Education» (episodio 4 de la temporada 4), y la respuesta a estos problemas siempre es el trabajo en equipo, la amistad y el crecimiento personal.

En 2019 y como parte de una campaña conjunta de autoaceptación para Cartoon Network y Dove, se publicó Your Magic Mind and Body, un libro virtual diseñado para leerlo en familia. La misma página de presentación del libro digital insta a les xadres a clicar un enlace «para obtener más información y herramientas y artículos detallados de expertos para desarrollar la confianza de tu hijo o hija en su cuerpo» (Tu cuerpo y mente son mágicos), lo que indica

\footnotetext{
${ }^{1}$ Dada la variedad de traducciones de los títulos y nombres de personajes, se conservan los nombres originales en inglés.
} 
que, aunque la serie Steven Universe también es popular entre personas adultas, la campaña es exclusivamente para niñes y jóvenes acompañades por sus xadres.

El libro en sí consiste en dos partes, tanto en su versión inglesa como en su traducción al español: la primera parte, que se puede saltar o rellenar por completo, es un cuestionario de personalización para la segunda parte, el libro en sí. Por ejemplo, una pregunta acerca del alimento favorito de le niñe se utiliza en el libro en la frase «Los humanos también necesitan combustible para crecer. ¡Como cuando [Nombre] come [alimento]!» (Sugar \& Danielsdottir 2019b: 9). En caso de saltar las preguntas, se omiten estas frases personalizadas, lo que resulta en un libro acerca de los seres humanos en general y no de le niñe en concreto. El libro habla, entre otros temas, sobre la alimentación y los pasatiempos, pero también sigue la línea de la serie original y habla de racismo («[nuestras enemigas] juzgan a todos por su color y su tipo. No permiten que nadie cree una nueva manera de ser.» Sugar \& Danielsdottir 2019b: 16) y las identidades no binarias (ibid. 17).

Ambas versiones, la original en inglés y la traducción al español, hablan en $1^{\mathrm{a}}, 2^{\mathrm{a}}$ y $3^{\mathrm{a}}$ persona: $1^{\text {a }}$ cuando habla directamente algún personaje («Me gusta dormir por diversión», «I like sleeping just for fun», Sugar \& Danielsdottir, 2019a, 201b: 6), 2a para hablarle a le lectore («iMe alegra verte!», «Good to see you!» ibid.: 2) y $3^{\mathrm{a}}$ para hablar tanto de las Gemas como de «los humanos», a quienes trata exclusivamente en masculino en español.

El inglés no tiene género gramatical, y los únicos pronombres tradicionales con marcas de género son «he» and «she», dos de los pronombres de la $3^{\text {a }}$ persona del singular. Gracias a esta característica del idioma, la versión original solo usa marcas de género para hablar de le lectore, y solo si le lectore decidió personalizar el libro para indicar sus pronombres. Sin embargo, la traducción al español utiliza el masculino genérico a lo largo del libro («los 
humanos», «los más jóvenes», «estamos juntos»), el femenino para las Gemas y, con dos excepciones puntuales que se explicarán a continuación, LNI para le lectore.

El error más importante de la traducción del libro se detecta durante la fase de personalización, en la sexta y última pregunta. Como ya se adelantó, existe la opción de no personalizar el libro, la cual resulta en la omisión de varias frases. Sin embargo, una vez se inicia la personalización, es obligatorio contestar a todas las preguntas para poder iniciar la lectura. Esta sexta pregunta reza, en inglés: «Oh! Let me introduce you to the Gems! Oh, I don't want to assume anything. If you don't mind me asking, what do you like to be called? You can choose more than one!» seguido de tres opciones: «he/him», «she/her»y «they/them». En efecto, se pueden escoger una, dos o tres de las opciones. Elegir más de una opción no parece dar resultados aleatorios, sino que aparentemente «they/them» es la primera prioridad, «she/her» la segunda y «he/him» la última, y se crea un libro con los pronombres elegidos que tengan la prioridad más alta. Metodología aparte, al presentar tres pronombres y habilitar la selección de varias opciones, el libro comparte un fuerte mensaje de aceptación y apoyo hacia las personas trans y no binarias y, en concreto, hacia las identidades fluidas y la exploración del género.

Sin embargo, la traducción española solo da dos opciones, «él» y «ella», acompañadas por la siguiente pregunta: « Oh! ¡Voy a presentarte a las Gemas! Pero no voy a dar nada por sentado. Si no te molesta mi pregunta, ¿con qué pronombre te identificas más? ¡Puedes elegir más de uno!» Resulta interesante no solo esta omisión de la tercera opción, que borra por completo a las personas no binarias, sino que la traducción introduce la palabra «pronombre» aunque no aparece en el original. El uso de «pronombre» contextos trans en español es un calco cultural, pues el problema principal en español son el género gramatical y los morfemas 
de género y no los pronombres como sucede en inglés. Este detalle añadido podría indicar cierto trabajo de documentación por parte del equipo de traducción que no se refleja en este borrado de identidades.

A pesar del carácter obligatorio de esta pregunta, una vez finalizada la etapa de personalización del libro solo se usa el género gramatical en tres ocasiones. Una de ellas es en la página 21 («iEstamos juntos en esto!») que, aunque podría excusarse como masculino genérico, debería de variar según el género elegido: esta frase la dicen las Gemas, que usan género gramatical femenino y deberían decir «juntas» si hablaran con una persona que use ese mismo género gramatical.

En la página 17, el libro habla de los roles de género y de las personas no binarias. Sin embargo, nos asigna una marca de género masculina: «Algunos humanos [creen] que tienes que actuar o verte de una manera determinada si eres niño, niña, o no binario» (sic). No queda claro si el equipo de traducción decidió usar «no binario» como masculino genérico o si simplemente no comprendieron lo que es una persona no binaria: aunque hay personas no binarias que utilizan el femenino, el masculino o una mezcla de las opciones disponibles en español, el término colectivo suele escribirse mediante LND o LNI: «si eres no binarie»o «si eres una persona no binaria», por ejemplo. Al incluir el término pero con el morfema incorrecto, se produce una situación de clara falta de comprensión por parte del equipo de traducción.

El tercer uso de género gramatical personal sucede en la página 19 («iEste es un libro sobre [pronombre] ahora!»). Ese pronombre es «ella»o «él» según se seleccionara durante la personalización, pero si no se personalizó el libro, la frase dice «un libro sobre ti». Esta es la única ocasión en la que se utiliza el género seleccionado al principio, pero la yuxtaposición 
de las tres versiones suscita más preguntas de las que responde: ¿por qué incorporar una única y artificiosa marca de género? ¿quiso justificar el equipo la presencia de la pregunta en el cuestionario? Al observar la traducción, esta parece la peor opción de las tres posibles, a saber: usar el género una única vez, eliminar la pregunta y esta única marca o conservar la pregunta y añadir más marcas. $^{2}$

Al revisar la traducción en su conjunto, aparecen dos posibilidades: o el equipo de traducción desconoce por completo la existencia de las personas no binarias — a pesar de que el libro habla de las personas no binarias y existe en un canon claramente queer y trans (Dunn 2016) - o el equipo tomó la decisión de ir completamente en contra del producto original para esconder a las personas no binarias bajo la alfombra. La primera posibilidad se podría haber subsanado mediante una documentación adecuada o la contratación de une traductore especializade o revisore de sensibilidad y se debe a la ignorancia, probablemente no malintencionada. La segunda posibilidad es más inquietante, pues elimina una parte importante no solo del texto sino de la identidad de su autore y de una parte importante de su público y constituiría un caso claro de binarismo y transfobia. Al tratarse de un proyecto de aceptación, esta exclusión indica que las personas no binarias no merecemos aceptación, respeto o siquiera nuestra inclusión en un libro escrito por una persona del colectivo.

\section{La traducción de terceres}

Una de las asimetrías más importantes en cuanto al género en español y en inglés es que, con la excepción de unas pocas unidades léxicas con género (boy, mother, pretty), solo se expresa el género en la $3^{\mathrm{a}}$ persona del singular. De hecho, en algunos contextos se puede utilizar ese

\footnotetext{
${ }^{2}$ Por ejemplo, «i[Esta/Este] es [Nombre], Gemas!» en lugar de «iLes presento a [Nombre], Gemas!» al finalizar el formulario de personalización.
} 
léxico para expresar ideas y no género. Por ejemplo, Parks y Barta muestran que la etiqueta mom friend (amiga mamá) va más allá del género para definir a las personas, del género que sean, que proporcionan cuidados feminizados a sus amigues, y que «se convierten en madres emocionales suplentes y en "amigas mamá”» (2018: 45, mi traducción). Sin embargo, con la excepción del LNI, en español se expresa el género del sujeto en $1^{\mathrm{a}}, 2^{\mathrm{a}}$ y $3^{\mathrm{a}}$ persona, tanto en singular como en plural.

Esta asimetría linguiística puede provocar desafíos traductores importantes. A modo ilustrativo, se repasarán las traducciones de varios personajes en tres series de televisión: Chilling Adventures of Sabrina y She-Ra, ambas producidas por Netflix, y Good Omens, producida por Amazon. Cada una de estas series cuenta con cuatro traducciones oficiales al español: el doblaje y subtitulado para América Latina y el doblaje y subtitulado para España. Al enfrentar estas cuatro versiones, podemos ver cómo se resuelven estos problemas traductores desde diferentes equipos de traducción, suponiendo que a los cuatro equipos se les impusieron las mismas pautas.

Empezando por Chilling Adventures of Sabrina, encontramos a Theo, un personaje trans representado por Lachlan Watson. En la primera parte de la serie ${ }^{3}$, Theo no expresa su género en inglés mientras que sus amigues y les adultes de su entorno le tratan en femenino. Al principio de la segunda parte, pide que le llamen «Theo» y que le traten en masculino. Varios de los monstruos y abusones de la serie le tratan en masculino, en femenino, o en una mezcla, como cuando un personaje del sexto episodio de la primera parte le dice «You're an abomination, boy-girl». Dado el contexto compartido en inglés (Watson es no binarie, la ausencia de género en $1^{\mathrm{a}}$ persona, varios momentos en los que Theo muestra incomodidad

\footnotetext{
${ }^{3}$ Netflix divide Chilling Adventures of Sabrina en «partes» y no en temporadas
} 
cuando le tratan en femenino), la comunidad de espectadores queer de la serie se dio cuenta de que Theo no era un personaje cis mucho antes del estreno de la segunda parte de la serie.

En las cuatro traducciones al español de esta serie se pierden la mayoría de las pistas sobre Theo a las que tienen acceso les espectadores anglófones pues Theo habla de sí mismo en femenino durante toda la primera parte, lo que resulta un comportamiento anómalo: Vacarezza (2018) documenta que las personas trans emplean el LNI y disimulan la pronunciación de los morfemas de género personal en las primeras fases de su transición social. En una de las traducciones, los subtítulos para España, Theo se refiere a sí mismo en femenino un par de escenas antes de decirles a sus amigues que le traten en masculino, lo que resultaría chocante en la vida real y aún más extraño en un formato reducido como es una serie: en lugar de horas antes, Theo se malgeneriza (se trata a sí mismo con el género equivocado) minutos antes de explicar quién es.

El problema que presenta Theo para la traducción no surge del LNI sino del hecho de que el inglés no tiene género gramatical y que es poco frecuente expresar género en $1^{\mathrm{a}}$ persona. Sin embargo, ese ejemplo ilustra de forma clara la invisibilización que puede surgir del uso del LNI, y en concreto su uso para ocultar la presencia de identidades no binarias. Aunque la traducción de Chilling Adventures of Sabrina no se haya hecho con el propósito de malgenerizar a Theo, el resultado final es que el público hispanohablante no recibe el mismo mensaje que el público anglófono.

Con gran probabilidad, el error se produjo porque el equipo de traducción, en la fase de comprensión (Hurtado Albir 2017: 328-330), no reparó en que esa ausencia de marcas era intencional, ató los cabos equivocados y, en la fase de reexpresión (ibid.), comunicó un mensaje diferente al original al añadir información que había sido deliberadamente omitida. 
Este mismo error puede producirse debido al LNI, con lo que su uso debe ser cuidadoso y concienzudo, teniendo en cuenta que la evasión de todas las marcas de género puede resultar en suposiciones incorrectas por parte de les receptores del mensaje.

Otro caso similar tuvo lugar unos meses más tarde del caso de Theo, en otra producción de Netflix. En octubre del 2019 se anunció que Jacob Tobia, autore y actore no binarie, se unía al elenco de She-Ra and The Princesses of Power para darle voz a un personaje no binario (Ramos 2019, Tobia 2019). Sin embargo, cuando se estrenó la temporada tres semanas después, muchas de las traducciones malgenerizaban a Double Trouble. En cuestión de días, las redes sociales se llenaron de comentarios acerca de varias de estas traducciones erróneas, entre ellas las traducciones al chino (@toad_witch 2019), al polaco (@roguemouse 2019), al portugués (@comunistasuave) y hasta al sueco, cuya traducción trata a Double Trouble en plural (@Sahrimnir 2019). El doblaje de España (@Miauschwitz 2019) y el subtitulado de América Latina (@Sarelllll 2019) le trataban en femenino, mientras que el doblaje de América Latina le trataba en masculino (@singingfer 2019). Tras los comentarios decepcionados del público, Netflix redobló o editó los subtítulos de escenas concretas para algunos mercados, con lo que incurrieron gastos adicionales por este error de traducción. Sin embargo, no se rectificaron los errores en todas las traducciones: actualmente, ambas traducciones para América Latina mantienen la malgenerización de Double Trouble, y es posible que otros idiomas también.

Una vez más, este error se introdujo por un error en la fase de descodificación y reexpresión del mensaje. Podría darse el caso de que los equipos de traducción no comprendieran el significado real del uso del pronombre «they» para Double Trouble o, como es frecuente en la traducción audiovisual, que se enviaran los episodios desordenados y les traductores 
empezaran por traducir episodios sin marcas de género, una vez más sin comprender la importancia de esas omisiones.

Los últimos dos ejemplos, los personajes de God y Pollution en Good Omens, son una buena representación tanto de los errores de malgenerización por falta de conciencia de género al traducir (un problema fácilmente solventable contratando a traductores especializades en lenguaje trans y no binario) como del posible uso del LNI para evitar malgenerizar a personas cuyo género se desconoce. En el libro por el mismo título en el que se basa Good Omens, Pollution es un hombre, pero en la serie es no binarie. Esto no se explicita más allá del uso puntual del pronombre "they" para hablar de elle. También se evitan todas las marcas de género para la figura de God (Dios), que resulta ser una mujer en el último episodio de la serie. Tres de las cuatro traducciones de Good Omens introducen errores al hablar tanto de Pollution como de God, mientras que una de ellas, el doblaje al español para España, traducida por Javier Pérez Alarcón, traduce bien a ambos personajes.

En su traducción, Pérez Alarcón utiliza LNI para Pollution porque detectó una ambigüedad en su presentación pero no pudo confirmar su género con el autor (Pérez Alarcón 2019). Pérez Alarcón traduce esta presentación, «This is Pollution» (episodio 6), como «He aquí a Polución». El uso del LNI en lugar del femenino o masculino fue la solución correcta, pues Neil Gaiman (2019) anunció en twitter que Pollution es no binarie mucho después de finalizar la traducción. Sin embargo, el subtitulado para España trata a Pollution en femenino («Ella es Polución»), los subtítulos para América Latina en masculino («Este es Polución») y el doblaje para América Latina como un objeto («Esto es Polución»).

La decisión de contratar a mujeres para representar a God no fue una coincidencia, sino que fue una decisión intencionada. A medida que se desarrolla la serie, les espectadores 
construyen su propia idea de cada personaje y, al no recibir información que indique que God usa el femenino, podrían asumir que usa el masculino como usa el Dios bíblico tradicionalmente. Durante los cinco primeros episodios, se le llama o «God»o «the Almighty» en todo momento en el que se usaría un pronombre en $3^{\mathrm{a}}$ persona, y no es hasta el último episodio que por fin se desvela su género («What if the Almighty planned it all along? [...] I wouldn't put it past her») y el público debe enfrentarse a sus propias conclusiones sesgadas.

Tanto el subtitulado para España como ambas traducciones para América Latina borran por completo este momento de reflexión. Estas tratan a God en masculino y usan varias palabras («el Señor», «el Poderoso», «el Todopoderoso») para hablar de ella, y al llegar al sexto episodio y la manifestación del género de God, el subtitulado para España borra las marcas de género («¿Y si Dios lo tenía todo planeado? [...] No me extrañaría») y ambas traducciones para América Latina reiteran el género masculino (ambas dicen « ¿Y si el Todopoderoso lo planeó así?»). En contraste, la traducción de Pérez Alarcón utiliza «su Omnipotencia» para mantener la ambigüedad original y conserva la revelación con « ¿Y si la omnipotente lo hubiera planeado así? [...] La veo muy capaz.»

Aunque, una vez más, el problema con tres de estas traducciones, en lo que a God se refiere, se origina en el uso del LNI en el original, la traducción de Pérez Alarcón también demuestra que el LNI se puede utilizar para mantener el mensaje de una manera mucho más acertada que asignar géneros binarios sin reflexión y sin cautela.

\section{Desafíos futuros del lenguaje no binario}


Aunque las traducciones de estas cuatro obras presentan retos y soluciones muy variadas, su combinación permite un análisis plural. El LNI es una estrategia tanto para la inclusión como para la exclusión, que puede evitar la malgenerización o invisibilizar tanto a las personas no binarias como a las mujeres. La interpretación del LNI depende, en gran parte, de le receptore: Koeser, Kuhn y Sczesny (2015) demuestran que las personas utilizan el LNI —al que ellas llaman «gender-fair language»— con más frecuencia si acaban de leer un texto que lo utilice y con menor frecuencia si han leído uno que utilice el masculino genérico. Koeser, Kuhn y Sczesny también hallaron que las mujeres son más receptivas al LNI y lo utilizan más que los hombres.

Hasta 2020 no se han realizado muchos estudios sobre el efecto de la exclusión de las personas no binarias mediante el uso intencionado del LNI. Sin embargo, podemos extrapolar de estudios relacionados con la expresión o supresión de identidades. Por ejemplo, Richeson y Nussbaum (2004) detallan cómo la comunicación «colorblind» (que no ve la raza) genera más sesgos racistas, tanto implícitos como explícitos, y Clark et al. (2018) señalan que les adolescentes no binaries postergan o descuidan sus citas médicas con mucha más frecuencia que les adolescentes binaries, probablemente debido de una manera u otra a una falta de capacitación sobre estas identidades entre el personal médico. Desde el contexto concreto de la aceptación y la educación, Gómez y Platero (2018: 113) dicen que «No poder concebir a las personas cuya identidad excede el modelo binario hegemónico implica un estado de vulnerabilidad social». Excusarse tras este desconocimiento para reproducir esta invisibilización solo contribuye al mismo desconocimiento de partida.

El constante cambio y desarrollo del lenguaje, junto al auge del lenguaje inclusivo en su sentido más amplio, resultan en amagos de comunicación bienintencionados pero que quedan 
cortos de alguna manera. Esto se ve en el uso irreflexivo del LNI, que puede servir para incluir a todas las personas minorizadas o marginalizadas por su género, pero también puede invisibilizar a estos mismos colectivos.

A través de los ejemplos prácticos analizados en este artículo, trato de poner tres puntos importantes de relieve.

Primero, el LNI es una herramienta útil cuando se desconoce el género de un sujeto o se quiere ocultar por alguna razón. Ya que el denominado masculino genérico no es realmente genérico, o por lo menos no lo es para todes les hablantes y en todos los contextos, es importante añadir otras estrategias a nuestra caja de herramientas no solo para la traducción sino para nuestra comunicación, tanto profesional como personal.

Segundo, la lectura irreflexiva del LNI, al igual que su uso, pueden provocar confusiones y ambigüedades. Estas no originan en la expresión del mensaje original, sino que dependen de los sesgos de le receptore. Este punto no debe entenderse como una amonestación contra su uso sino como una advertencia.

Por último, el uso exclusivo del LNI para hablar de personas que utilizan el LND, o para ocultar la presencia de personas no binarias, es un acto de invisibilización y de binarismo. El problema, como se ha explicado, no yace en el uso del LNI sino en el rechazo a otras estrategias que permitan visibilizar a este colectivo minorizado. Al fin y al cabo, todo el mundo tiene derecho a su identidad, a la representación y visibilidad a través del lenguaje y al trato respetuoso de su comunidad.

\section{Bibliografía}

Clark, Beth A., Veale, Jaimie F., Townsend, Marria, Frohard-Dourlent, Hélène y Saewyc, Elizabeth (2018). Non-binary youth: Access to gender-affirming primary health care. 
International Journal of Transgenderism. 19(2): 158-169. DOI: 10.1080/15532739.2017.1394954

Dunn, Eli (2016). Steven Universe, fusion magic, and the queer cartoon carnivalesque. Gender Forum. 56: pp 44-57.

Ermac, Raffy (2019). Cartoon Network Confirmed This Steven Universe Character Is Intersex. En Pride.com. 26 de junio del 2019. $<$ https://www.pride.com/geek/2019/6/26/cartoon-network-confirmed-steven-universecharacter-intersex $>$

Gaiman, Neil (2019). «Our glorious and non binary Pollution... \#GoodOmens» [Publicación $\begin{array}{llllll}\text { en } & \text { Twitter, } & 14 & \text { de } & \text { noviembre } & \text { del }\end{array}$ <https://twitter.com/neilhimself/status/1124778930207109120>

Gilchrist, Tracy E. (2017). Virginia GOP Devises Transphobic Way to Refuse to Call Danica Roem 'Gentlewoman'. En The Advocate. 23 de noviembre del 2017. <https://www.advocate.com/politics/2017/11/23/virginia-gop-devises-transphobic-wayrefuse-call-danica-roem-gentlewoman>

Hurtado Albir, Amparo (2017). Traducción y Traductología. Madrid: Cátedra.

Koeser, Sara, Kuhn, Elisabeth A., \& Sczesny, Sabine (2015). Just Reading? How GenderFair Language Triggers Readers' Use of Gender-Fair Forms. Journal of Language and Social Psychology, 34(3), 343-357. 10.1177/0261927X14561119

López Gómez, Isabel y R. Lucas Platero (2018). Faltan palabras! Las personas no binarias en el Estado español. ex aequo - Revista da Associação Portuguesa de Estudos sobre as Mulheres. 38: 111-127. doi:10.22355/exaequo.2018.38.08

López, Ártemis (2019a). Syd-nificant others or Syd-nificant selves? Audiovisual translation of gender identities for mainstream audiences. (Comunicación en el congreso the They, Herself, Em and You en Queen's University, Kingston, ON, el 11 de junio del 2019)

López, Ártemis (2019b). Tú, yo, elle y el lenguaje no binario. La Linterna del Traductor. 19: 142-150. <http://www.lalinternadeltraductor.org/n19/traducir-lenguaje-no-binario.html>

Los Replicantes (2020). El artículo transfóbico del diario ABC que ha generado críticas en redes sociales. En Los Replicantes. 4 de octubre del 2020. $<$ https://www.losreplicantes.com/articulos/articulo-transfobico-abc-criticas-redessociales/>

Parks, Elizabeth S. y Kristen Barta (2018). Are You My Mother? Perpetuating Gender Inequality through Listening Expectations and Relational Roles. Journal of Research in Gender Studies 8(1). 28-48.

Pérez Alarcón, Javier (2019). Romper el código binario: elle en la traducción para doblaje. Facultat de Traducció i Interpretació, Universitat Autònoma de Barcelona. 25 de agosto del 2020. <https://youtu.be/zkf5EM14h2w?t=2664> 
Ramos, Dino-Ray (2019). Jacob Tobia Joins 'She-Ra And The Princesses Of Power' As NonBinary Character. En Deadline. [21 de octubre del 2019] $<$ https://deadline.com/2019/10/jacob-tobia-she-ra-and-the-princesses-of-power-non-binarydouble-trouble-lgbtq-netflix-1202765255/>

Richeson, Jennifer. A., \& Nussbaum, Richard. J. (2004). The impact of multiculturalism versus color-blindness on racial bias. Journal of Experimental Social Psychology, 40(3), 417-423. DOI: 10.1016/j.jesp.2003.09.002

Serbeto, Enrique (2020). Bélgica nombra al primer ministro transexual de Europa. En ABC. 3 de octubre del 2020. <https://www.abc.es/internacional/abci-belgica-nombra-primerministro-transexual-europa-202010022008_noticia.html >

Sugar, Rebeca y Sigrun Danielsdottir (2019a). Your Magic Mind and Body. <https://www.stevenuniverseselfesteem.com/storybook.htmlhtml>

Sugar, Rebeca y Sigrun Danielsdottir (2019b). Tu cuerpo y tu mente son mágicos. <https://www.stevenuniverseselfesteem.com/es/storybook.html>

Tobia, Jacob (2019). Anuncio de vídeo para Netflix en @most. 21 de octubre del 2019. <https://www.instagram.com/p/B35CajlAo-j >

Tu cuerpo y mente son mágicos (2019). Steven Universe and the Dove Self-Esteem Project. Cartoon Network, Dove y Steven Universe. <https://www.stevenuniverseselfesteem.com/es/index.html>

Vacarezza, Nayla Luz (2018). Decir el propio género. Feminidades, usos del género gramatical y nombre propio. Cadernos Pagu 52, DOI: 10.1590/18094449201800520010

@ comunistasuave (2019). «@comunistasuave: Me incomodou um pouco em She-ra q nada indicou q o Double Trouble é nb, os pronomes na dublagem foram masculinos. Alguém q viu em inglês, foi assim tb?» [Publicación en Twitter, 14 de noviembre del 2019] <https://twitter.com/comunistasuave/status/1195083426220716039>

@Miauschwitz (2019). «@Miauschwitz: He puesto el doblaje en castellano: traducen a Double Trouble como "Dupla" y le tratan en femenino. Gracias @ NetflixES por destruir el diminuto trocito de representación NB que tenías.» [Publicación en Twitter, 6 de noviembre del 2019] <https://twitter.com/Miauschwitz/status/1192022099948441602>

@ roguemouse (2019). «@ roguemouse: I don't know whose decision it was to assign the nonbinary Double Trouble in \#sheraseason4 a male gender, male pronouns and male name ("Trouble" as a surname) in the Polish translation, but this goes against the very idea of \#nonbinary representation.\#SheRa @NetflixPL @ netflix» [Publicación en Twitter, 6 de noviembre del 2019] <https://mobile.twitter.com/roguemouse/status/1192010937101934592>

@ Sahrimnir (2019). Re: Erasing Double-Trouble in Translations. [Publicación en Reddit, 8 de noviembre del 2019] 
<https://www.reddit.com/r/PrincessesOfPower/comments/dt1li8/erasing_doubletrouble_in_ translations/f6uxn5o/>

@Sarelllll (2019).«@Sarelllll: En latino los subs le trataban en femenino también, y en el doblaje creo que le tratan en masculino, pero mantuvieron Double Trouble como nombre» [Publicación en Twitter, $10 \quad$ de diciembre del 2019] <https://twitter.com/Sarelllll/status/1204394805931003904>

@ singingfer (2019). «@ singingfer: Hilo de veces que Double Trouble sufre misgender en el doblaje latino de She-ra:» [Publicación en Twitter, 20 de noviembre del 2019] <https://twitter.com/singingfer/status/1200599041043681281>

@ toad_witch (2019). Re: Erasing Double-Trouble in Translations. [Publicación en Reddit, 7 de noviembre del 2019] <https://www.reddit.com/r/PrincessesOfPower/comments/dt1li8/erasing_doubletrouble_in_ translations/f6upip2/> 